Research Article

\title{
Iterative Dipole Moment Method for the Dielectrophoretic Particle-Particle Interaction in a DC Electric Field
}

\author{
Qing Zhang ${ }^{1}$ and Kai Zhang $\mathbb{D}^{2}$ \\ ${ }^{1}$ China Tobacco Standardization Research Center, Zhengzhou, China \\ ${ }^{2}$ Institute of Fluid Engineering of China Jiliang University, Hangzhou, China \\ Correspondence should be addressed to Kai Zhang; zkzb3026@yahoo.com
}

Received 1 February 2018; Accepted 12 March 2018; Published 9 May 2018

Academic Editor: Martin Seipenbusch

Copyright ( 2018 Qing Zhang and Kai Zhang. This is an open access article distributed under the Creative Commons Attribution License, which permits unrestricted use, distribution, and reproduction in any medium, provided the original work is properly cited.

\begin{abstract}
Electric force is the most popular technique for bioparticle transportation and manipulation in microfluidic systems. In this paper, the iterative dipole moment (IDM) method was used to calculate the dielectrophoretic (DEP) forces of particle-particle interactions in a two-dimensional DC electric field, and the Lagrangian method was used to solve the transportation of particles. It was found that the DEP properties and whether the connection line between initial positions of particles perpendicular or parallel to the electric field greatly affect the chain patterns. In addition, the dependence of the DEP particle interaction upon the particle diameters, initial particle positions, and the DEP properties have been studied in detail. The conclusions are advantageous in elelctrokinetic microfluidic systems where it may be desirable to control, manipulate, and assemble bioparticles.
\end{abstract}

\section{Introduction}

Microfluidic systems are widely used for biochemical analysis over the past decade, and electric forces are often used as an efficient and effective transport mechanism, which does not involve the intervention of moving parts and offers good manipulation over sample handling. Deposition of bioparticles and colloids onto the surface [1] is very important for many biochemical processes, such as assembly of carbon nanotubes $[2,3]$ and trapping of nanoparticles and nanoparticle synthesis [4-6]. In these processes, particles' translation and rotation should be controlled accurately for counting and assembly of these particles [7, 8]. In microfluidic systems, electrical and hydrodynamic forces [9] dominate particles' transportation. In the bulk fluid, particle transport is mainly affected by the hydrodynamic interactions and its neighboring particles.

As one of the important forces of particle manipulation, dielectrophoretic (DEP) force has been drawing much attention in recent years. Equivalent dipole moment (EDM) has usually been used to calculate DEP force for single particle but cannot be used for the interaction between neighboring particles in an electric field. Maxwell stress tensor (MST) method [10] is complicated for the calculation of a large number of particles' interaction [11]. The iterative dipole moment (IDM) [12-14] method, which does not solve complicated differential equations of an electric field while the particle is moving, was used in this work for calculating the DEP forces of particle interactions.

To control the assembling of bioparticles with high accuracy, an in-depth study is necessary to the underlying mechanisms of DEP particle-particle interaction in microfluidic systems. The impetus for the present study came from the importance in understanding the mechanics of particle assembling, which is often used to produce specialized material in a lab on a chip. In the present study, the DEP interaction of particle-particle [15] in a DC electric field will be studied, and a mathematical model based on the Lagrangian method incorporating DEP particle-particle interactions [16] will be presented to compute particle trajectories, and 


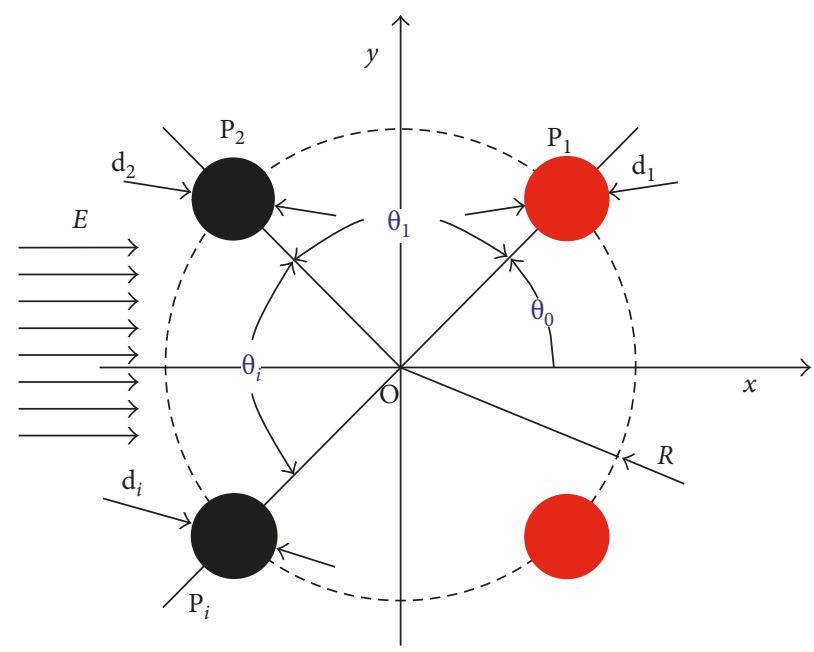

Figure 1: Cylindrical particles are equispaced on a circle in a two-dimensional DC uniform electric field $\mathbf{E}=1 \mathrm{KV} / \mathrm{m}$; here, red represents positive DEP and black represents negative DEP.

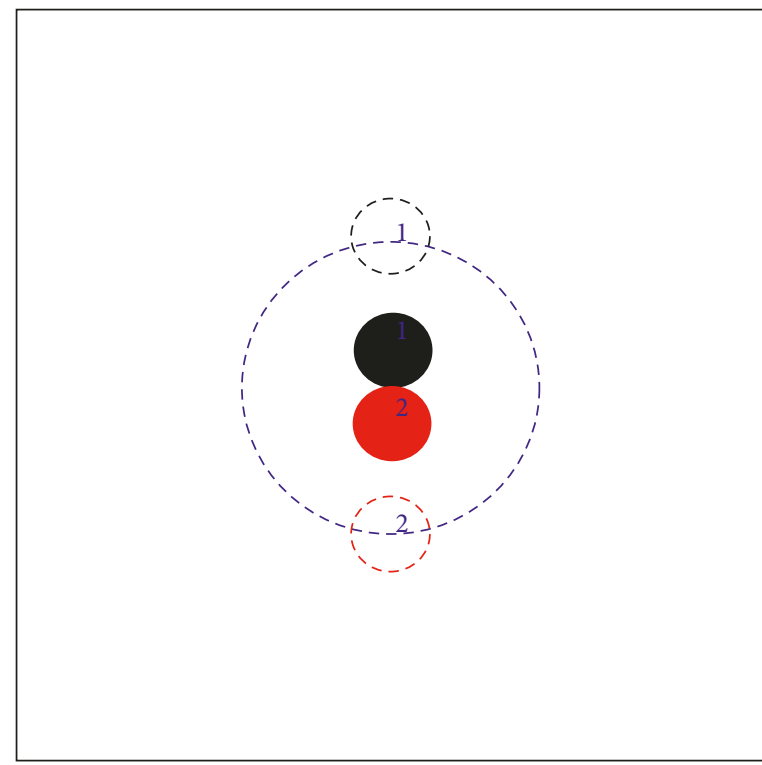

(a)

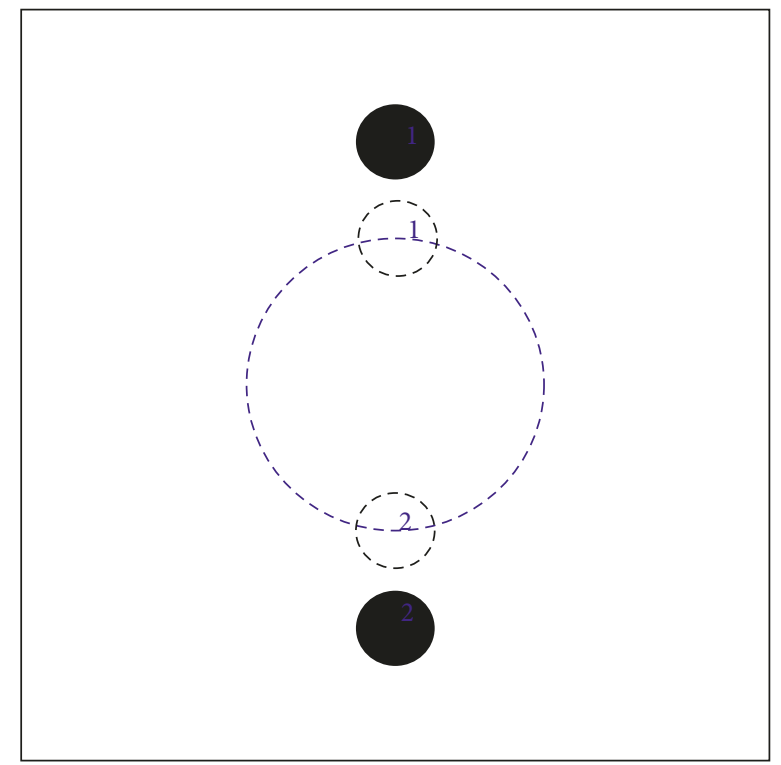

(b)

Figure 2: Two particles (red or black dashed line) are initially equispaced on a circle (blue dashed line) with $\theta_{0}=\pi / 2$ in a uniform DC electric field with $E=1 \mathrm{kV} / \mathrm{m}$ along $x$ direction, and then the final particle chains (solid particles) are, respectively, shown after DEP interaction under different statuses: (a) two negative (black) DEP particle, (b) one positive (red) and one negative DEP particle.

consequently, the assembling will be calculated. Finally, the dependence of particle assembling on the particle's initial position and radius will be further concluded and discussed in detail.

\section{Computational Model}

As shown in Figure 1, in a two-dimensional incompressible still media, DEP cylindrical particles are initially equispaced on a circle with radius $R=20 \mu \mathrm{m}$ and an initial angle $\theta_{0}$ with respect to the applied electric field. Here, particle diameter $d=5 \mu \mathrm{m}$, for negative DEP, the permittivity of the media and particles are $\varepsilon_{m}=6.9 e-10 \mathrm{CV}^{-1} \cdot \mathrm{m}^{-1}$ and $\varepsilon_{p}=2.5 * 8.8541878176 e-12 \mathrm{CV}^{-1} \cdot \mathrm{m}^{-1}$; for positive dielectrophoresis, $\varepsilon_{m}=2.5 * 8.8541878176 e-12 \mathrm{CV}^{-1} \cdot \mathrm{m}^{-1}$ and $\varepsilon_{p}=6.9 e-10 \mathrm{CV}^{-1} \cdot \mathrm{m}^{-1}$.

The dielectrophoretic force, $\mathbf{F}_{\mathrm{DEP}}$, acting on a spherical, homogeneous particle suspended in a local electric field gradient is given by the expression

$$
\mathbf{F}_{\mathrm{DEP}}=2 \pi r_{p}^{3} \varepsilon_{m} \operatorname{Re}[K(w)] \nabla \mathbf{E}^{2},
$$

where $r_{p}$ is the particle radius, $\varepsilon_{m}$ is the permittivity of the suspending medium, $\nabla$ is the Del vector (gradient) operator, $\mathbf{E}$ is the electric field incorporating additional fields due to particle interactions, and $\operatorname{Re}[K(w)]$ is the real part of the Clausius-Mossotti factor, which is given by 


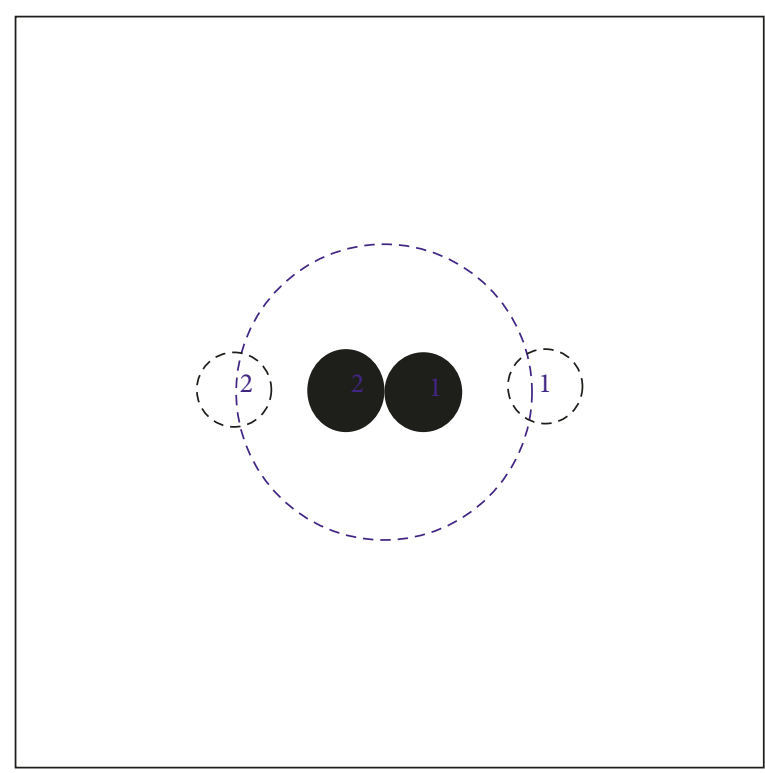

(a)

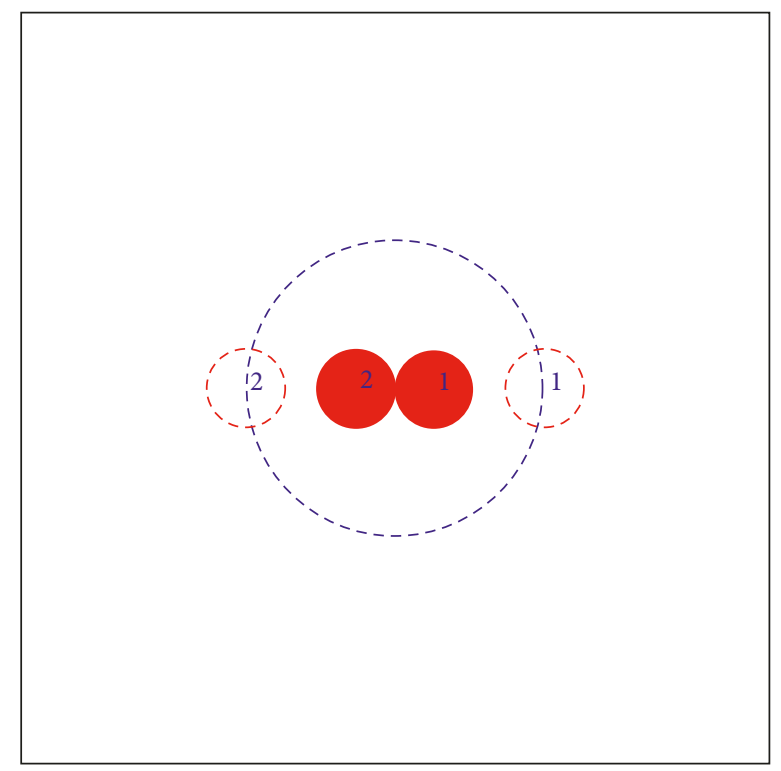

(b)

(c)

FIgURE 3: The initial position of two particles are initially equispaced on a circle with $\theta_{0}=0$ in a uniform DC electric field with $E=1 \mathrm{kV} / \mathrm{m}$ along $x$ direction, and the final particle chains after DEP interaction under different status: (a) two negative DEP particle, (b) two positive DEP particle, and (c) one positive and one negative DEP particle.

$$
K(w)=\frac{\left(\varepsilon_{p}^{*}-\varepsilon_{m}^{*}\right)}{\left(\varepsilon_{p}^{*}+2 \varepsilon_{m}^{*}\right)},
$$

And the dielectrophoretic force can be modified as follows:

$$
\mathbf{F}_{\mathrm{DEP}}=4 \pi r_{p}^{3} \varepsilon_{m} \frac{\left(\varepsilon_{p}-\varepsilon_{m}\right)}{\left(\varepsilon_{p}+2 \varepsilon_{m}\right)} \mathbf{E} \cdot \nabla \mathbf{E}
$$

medium and particle, respectively, and $\varepsilon^{*}=\varepsilon-(j \sigma / w)$ with $\sigma$ is the conductivity, $\varepsilon$ is the permittivity, and $w$ is the angular frequency of the applied electric field. The limiting direct current (DC) case of the equation is

$$
K(w=0)=\frac{\left(\varepsilon_{p}-\varepsilon_{m}\right)}{\left(\varepsilon_{p}+2 \varepsilon_{m}\right)} .
$$

Considering the influence of the dipole-induced field of other particles surrounding particle $i$, the modified electric field near the particle located at $\mathbf{r}_{i}$ is shown below:

$$
E_{i}^{(1)}(r)=E_{0}(r)+\sum_{j=1, j \neq i}^{n} E_{d}^{(0)}\left(r_{j}, r\right), \quad i=1,2,3, \ldots, N,
$$




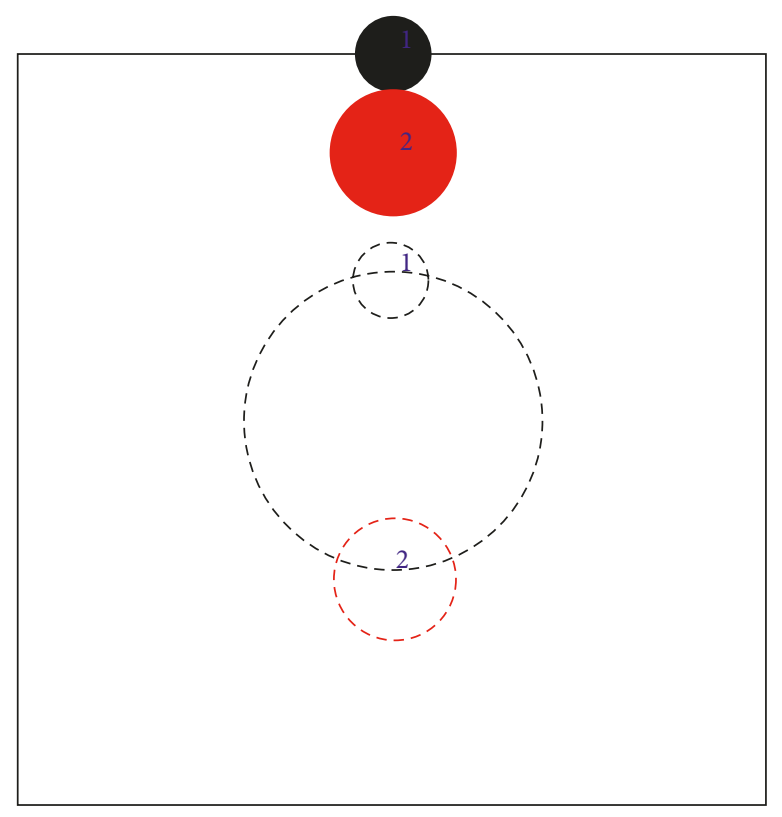

(a)

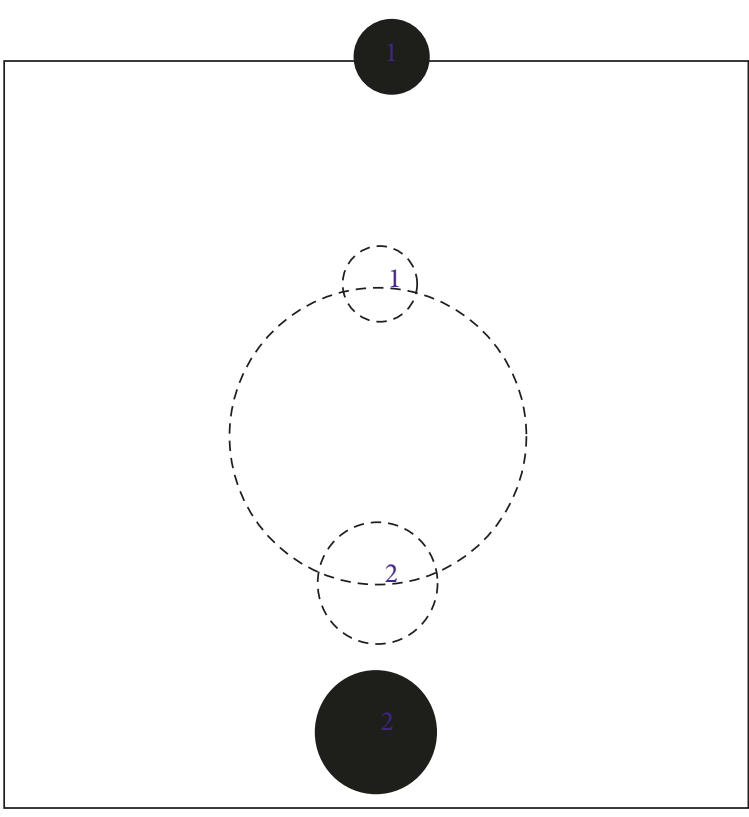

(b)

FIgURE 4: The initial position of two particles is initially equispaced on a circle with $\theta_{0}=\pi / 2$ in a uniform DC electric field with $E=1 \mathrm{kV} / \mathrm{m}$ along $x$ direction, and the final particle chains after DEP interaction under different status: (a) two heterogeneous particles with different diameters, (b) two negative DEP particles with different diameters.

where $E_{i}^{(1)}(r)$ denotes the modified electric field and $E_{d}^{(0)}\left(r_{j}, r\right)$ is the influence of the dipole-induced field of particle $j$; here,

$$
\begin{aligned}
\left(E_{d}\right)_{x} & =-\frac{\partial \varphi_{d}}{\partial x} \\
& =-\frac{\left|P_{i}\right|}{2 \pi \varepsilon_{m}}\left[\frac{\cos \alpha_{i}}{\Delta x_{i}^{2}+\Delta y_{i}^{2}}-\frac{2 \Delta x_{i}\left(\Delta x_{i} \cos \alpha_{i}+\Delta y_{i} \sin \alpha_{i}\right)}{\left(\Delta x_{i}^{2}+\Delta y_{i}^{2}\right)^{2}}\right], \\
\left(E_{d}\right)_{y} & =-\frac{\partial \varphi_{d}}{\partial y} \\
& =-\frac{\left|P_{i}\right|}{2 \pi \varepsilon_{0}}\left[\frac{\sin \alpha_{i}}{\Delta x_{i}^{2}+\Delta y_{i}^{2}}-\frac{2 \Delta y_{i}\left(\Delta x_{i} \cos \alpha_{i}+\Delta y_{i} \sin \alpha_{i}\right)}{\left(\Delta x_{i}^{2}+\Delta y_{i}^{2}\right)^{2}}\right] .
\end{aligned}
$$

The modified electric field induces a new dipole moment, which again induces a new electric field as follows:

$$
E_{i}^{(1)}\left(r_{i}\right) \rightarrow P_{i}^{(1)} \rightarrow E_{d}^{(1)}\left(r_{i}, r\right), \quad i=1,2,3, \ldots, N,
$$

where $E_{i}^{(1)}\left(r_{i}\right), P_{i}^{(1)}$, and $E_{d}^{(1)}\left(r_{i}, r\right)$ are the modified electric field, the modified dipole moment, and the modified dipoleinduced electric field, respectively. These parameters can be iteratively modified until a converged value of the electric field $E_{i}^{(n)}\left(r_{i}\right)$ is obtained and the final dipole moment considering other particles' influence can be achieved:

$$
P_{i}=\frac{4 \pi a_{i}^{3} \varepsilon_{m}\left(\varepsilon_{i}-\varepsilon_{m}\right)}{\varepsilon_{i}+2 \varepsilon_{m}} E_{i}^{(n)}\left(r_{i}\right) .
$$

These particles are assumed to be far from boundaries. The forces on particles are calculated by (1) while the dipole moment have been modified using IDM every time step, and particle transportation can be easily solved for particle trajectories and final particle chains.

\section{Results and Discussion}

Imagine a particle suspended in a dielectric fluid and subjected to a uniform electric field, which will polarize the dielectric particle and induce a dipole moment in it, then the dipole moment will induce an electrostatic potential as shown below:

$$
\varphi_{d}=\frac{P_{i} \cos \theta}{4 \pi \varepsilon_{m} r^{2}} .
$$

When particles are close to each other, one particle's induced electrostatic potential will distort its neighbor's electric field and make it nonuniform, and then the DEP force acting on its neighbor comes to a nonequilibrium state. The nonzero DEP forces on particles influencing each other suspended in a two-dimensional DC uniform electric field can be calculated by the IDM method.

As shown in Figure 2, if two heterogeneous DEP particles with same diameter are released at a small distance from each other and perpendicular to the electric field, they will attract each other and are likely to cluster at the center of line of their initial position. However, if all parameters except for the DEP property are the same, the results show that two homogeneous DEP particles will repel each other to move outwards symmetrically. 


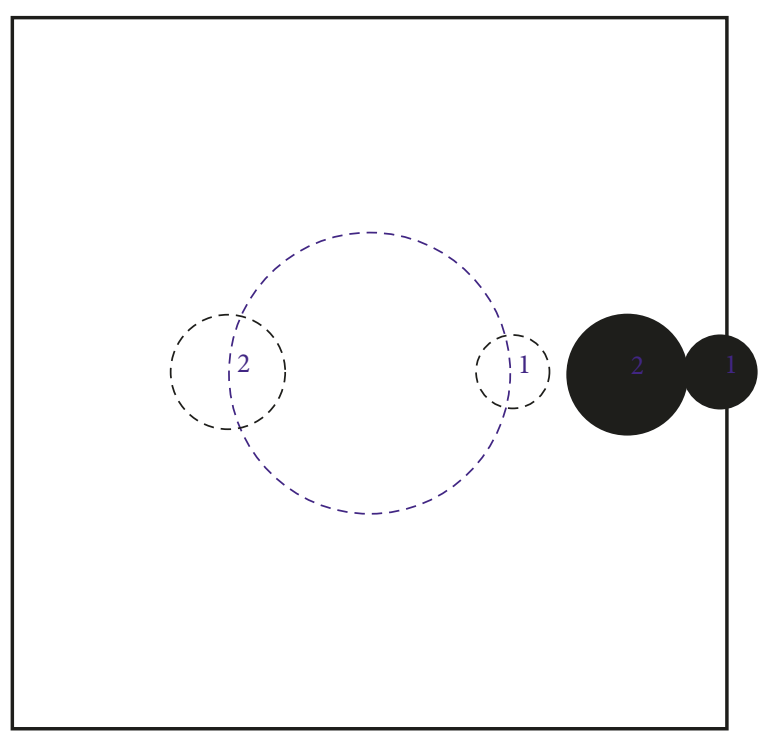

(a)

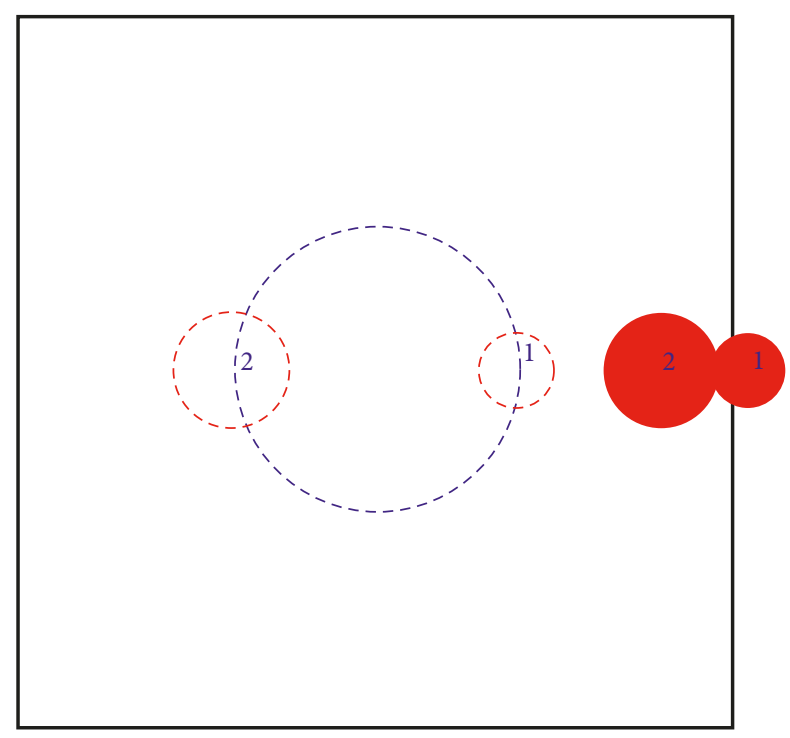

(b)

FIGURE 5: The initial position of two particles is initially equispaced on a circle with $\theta_{0}=0$ in a uniform DC electric field with $E=1 \mathrm{kV} / \mathrm{m}$ along $x$ direction, and the final particle chains after DEP interaction under different status: (a) two negative DEP particle with different diameter and (b) two positive DEP particle with different diameters.

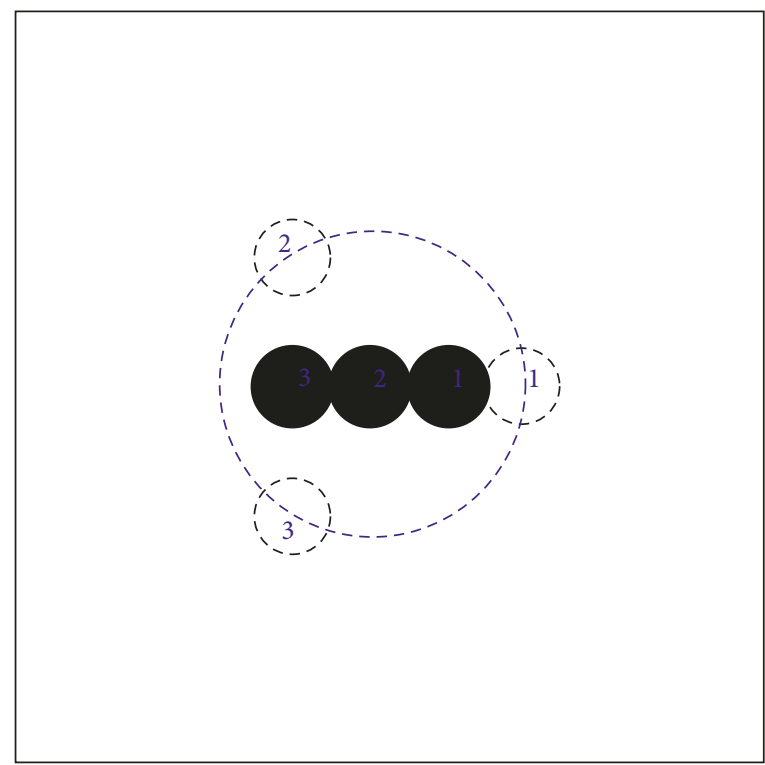

(a)

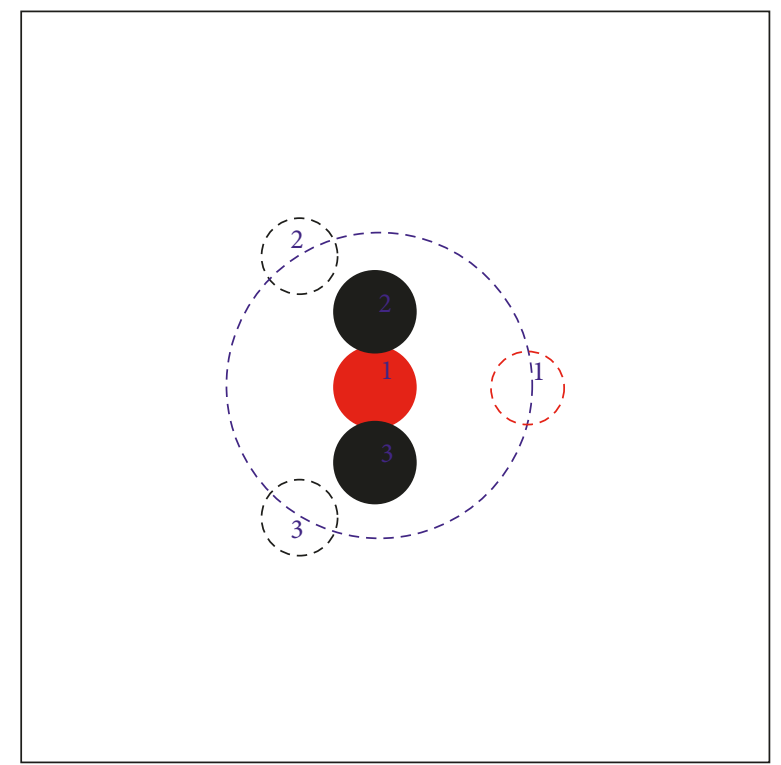

(b)

Figure 6: The initial position of three particles is initially equispaced on a circle with $\theta_{0}=0$ in a uniform DC electric field with $E=1 \mathrm{kV} / \mathrm{m}$ along $x$ direction, and the final particle chains after DEP interaction under different status: (a) three negative DEP particle and (b) two negative and one positive DEP particle.

From Figure 3, it can be seen that if two homogeneous DEP particles with same diameter are released at a small distance from each other and parallel to the electric field, they will attract each other and cluster at the center of line of their initial position. However, if all parameters except for the DEP property are the same, the results show that two heterogeneous DEP particles will repel each other to move outwards symmetrically.
Figure 4 shows the DEP interaction between two particles with different diameters. Figure 4(a) shows that when two heterogeneous DEP particles are released at a small distance from each other and perpendicular to the electric field, they will attract and cluster. However, as the difference of two particles' diameters becomes larger, the position of final particle chains deviates from the center of line of their initial position, and the chains will move towards the side of 


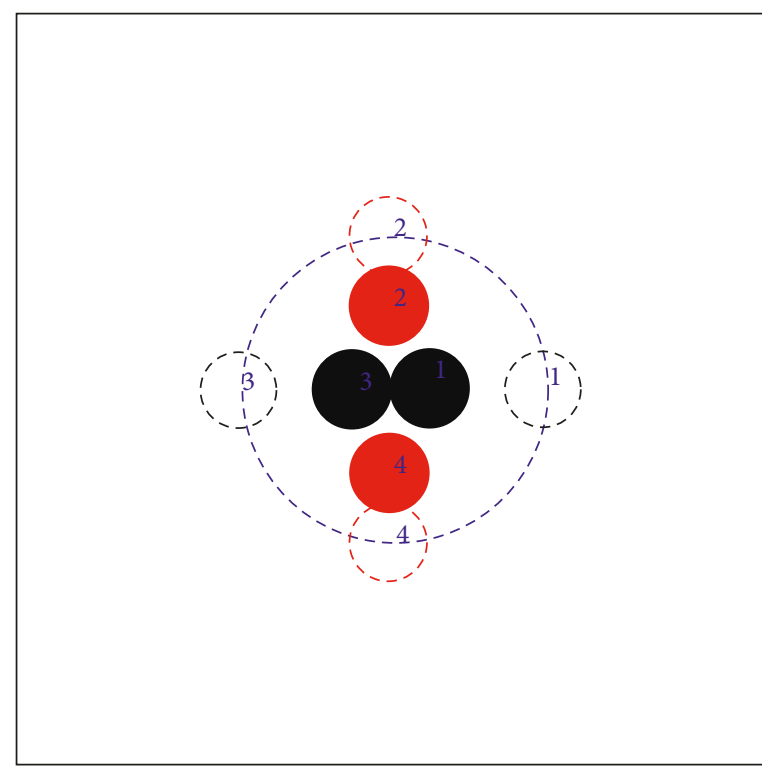

(a)

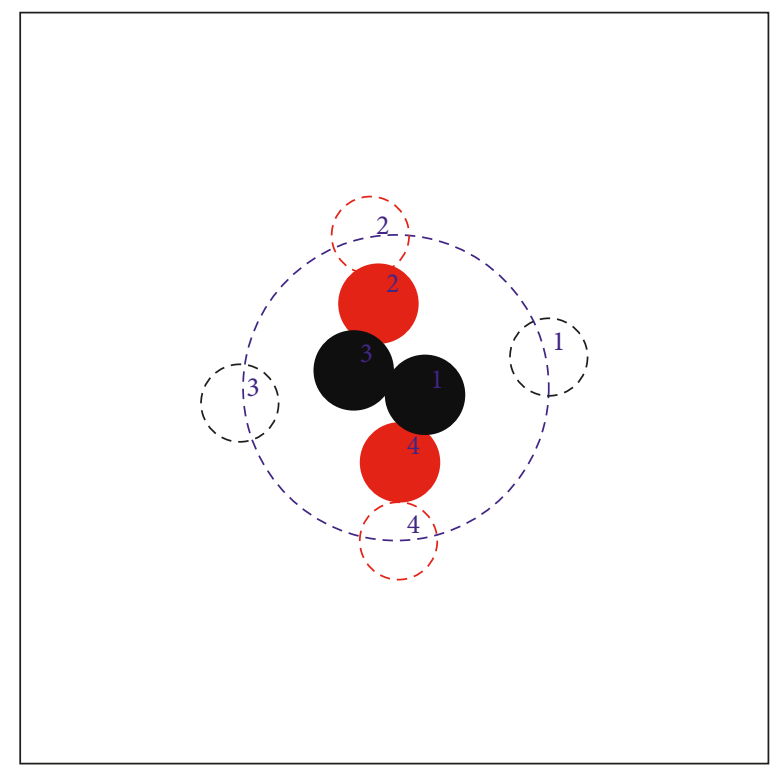

(b)

FIgURE 7: The initial position of two negative and two positive DEP particles are initially equispaced on a circle in a uniform DC electric field with $E=1 \mathrm{kV} / \mathrm{m}$ along $x$ direction, and the final particle chains after DEP interaction under different status: (a) $\theta_{0}=0$, (b) $\theta_{0}=\pi / 16$.

the smaller particle. In addition, if all parameters except for the DEP property are the same, Figure $4(\mathrm{~b})$ shows that the two homogeneous DEP particles with different diameters will repel each other to move outwards asymmetrically, and the smaller particle moves faster.

Figure 5 shows that when two homogeneous DEP particles with different diameters are released at a small distance from each other and parallel to the electric field, they will attract and cluster, and the chains will move towards the side of the smaller particle.

From Figure 6(a), it can be seen that if three homogeneous dielectrophoretic particles with same diameters are initially equispaced on a small circle, they will attract each other and finally cluster parallel to the electric field. However, from Figure 6(b), it can found that when one of the particles changed its DEP property, they still clustered but the chains were perpendicular to the electric field.

From Figure 7(a), it can be seen that due to the symmetric configuration, homogeneous DEP particles 1 and 3 are attracted to move inwards because their connection line is parallel to the electric field; at the same time, positive DEP particles 2 and 4 are attracted by the chains of negative DEP particles 1 and 3 to move inward. While the connection line of the initial position of homogeneous particles 2 and 4 is perpendicular to the electric field, they cannot contact the chains of negative DEP particles 1 and 3. However, when the connection line between two homogeneous DEP particles 2 and 4 has a little shift $\theta_{0}=\pi / 16$ from the line perpendicular to the electric field, DEP interactions among particles cause four particles to cluster as shown in Figure 7(b).

As shown in Figure 8, three negative and two positive DEP particles are initially equispaced on a circle with $\theta_{0}=0$ in a uniform DC electric field. From the above conclusions, it can be found that the homogeneous adjacent particles whose

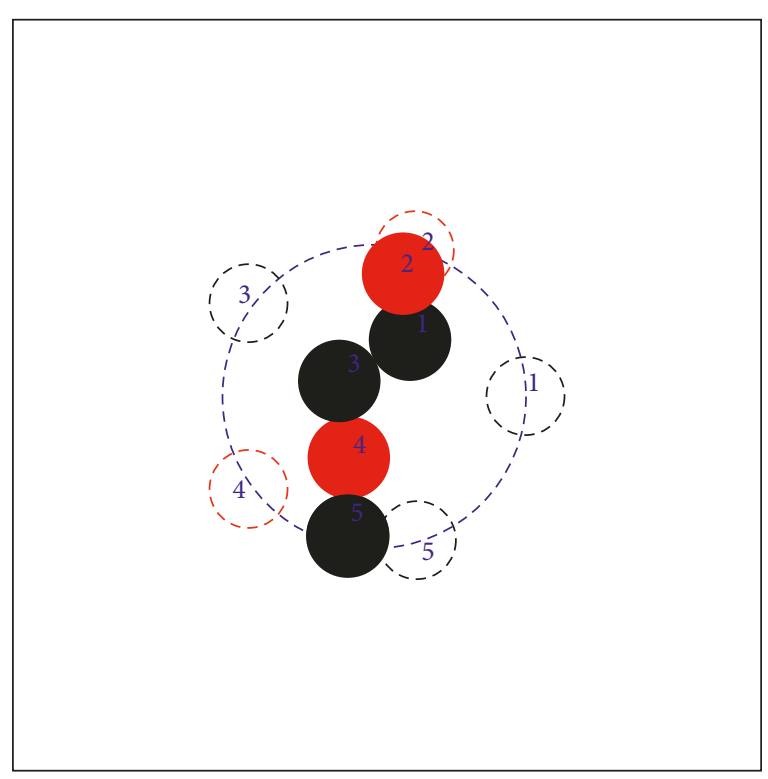

FIGURE 8: The initial position of five heterogeneous DEP particles is initially equispaced on a circle with $\theta_{0}=0$ in a uniform DC electric field with $E=1 \mathrm{kV} / \mathrm{m}$ along $x$ direction, and the final particle chains after DEP interaction.

connection line is not perpendicular to the electric field will cluster parallel to the electric field, and homogeneous adjacent heterogeneous particles will cluster perpendicular to the electric field, and eventually form the complex and asymmetric structure as shown in Figure 8.

According to these aforementioned conclusions, the regular aggregation patterns of large number of polarizable particles can also be well understood and can be used to control, manipulate, and assemble polarizable particles. 


\section{Conclusions}

The IDM method was used to study multiple particle DEP interactions in a uniform DC electric field, and the DEP interactions among particles cause particles to cluster. It was found that the homogeneous adjacent particles whose connection line is not perpendicular to the electric field will cluster parallel to the electric field, and homogeneous adjacent heterogeneous particles will cluster perpendicular to the electric field; however, as the difference of two particles' diameters becomes larger, the position of final particle chains deviates from the center of line of their initial position, and the chains will move towards the side of the smaller particle. In addition, the dependence of the DEP particle interaction upon the particle diameters, initial particle positions, and the DEP properties have been studied in detail. This can be advantageous in elelctrokinetic microfluidic systems where it may be desirable to control, manipulate, and assemble cylindrical bioparticles.

\section{Conflicts of Interest}

The authors declare that there are no conflicts of interest regarding the publication of this paper.

\section{Funding}

The authors received funding from the National Natural Science Foundation of China (11472260).

\section{References}

[1] W. J. Tian, J. P. Huang, and K. W. Yu, "Electric-field-induced interaction between biological cells or colloidal particles," Journal of Applied Physics, vol. 105, no. 10, p. 10989, 2009.

[2] R. M. Towshif, C. F. Schmidt, and R. Alexandra, "Single-walled carbon nanotubes probed with insulator-based dielectrophoresis," Analytical Chemistry, vol. 89, no. 24, pp. 13235-13244, 2017.

[3] Z. M. Asif, P. Punnag, H. Paul et al., "Dielectrophoresisassisted plasmonic trapping of dielectric nanoparticles," Physical Review A, vol. 95, no. 2, p. 023840, 2017.

[4] M.-Z. Yu, J.-Z. Lin, and T.-L. Chan, "Effect of precursor loading on non-spherical $\mathrm{TiO}_{2}$ nanoparticle synthesis in a diffusion flame reactor," Chemical Engineering Science, vol. 63, no. 9, pp. 2317-2329, 2008.

[5] M. Yu, Y. Liu, J. Lin, and M. Seipenbusch, "Generalized TEMOM scheme for solving the population balance equation," Aerosol Science Technology, vol. 49, no. 11, p. 1021, 2015.

[6] M. Yu, J. Lin, and T. Chan, "Numerical simulation of nanoparticle synthesis in diffusion flame reactor," Powder Technology, vol. 181, no. 1, pp. 9-20, 2008.

[7] J. Lin, X. Pan, Z. Yin, and X. Ku, "Solution of general dynamic equation for nanoparticles in turbulent flow considering fluctuating coagulation," Applied Mathematics and Mechanics, vol. 37, no. 10, pp. 1275-1288, 2016.

[8] J. Lin, Y. Wang, P. Zhang, and X. Ku, "Mixing and orientation behaviors of cylindrical particles in a mixing layer of an Oldroyd-B fluid," Chemical Engineering Science, vol. 176, pp. 270-284, 2018.
[9] D. Das and D. Saintillan, "Electrohydrodynamic interaction of spherical particles under Quincke rotation," Physical Review E, vol. 87, no. 4, p. 043014, 2013.

[10] S. Kumar and P. J. Hesketh, "Interpretation of ac dielectrophoretic behavior of tin oxide nanobelts using Maxwell stress tensor approach modeling," Sensors and Actuators B: Chemical, vol. 161, no. 1, pp. 1198-1208, 2012.

[11] G. Liu, J. S. Marshall, S. Q. Li, and Q. Yao, "Discrete-element method for particle capture by a body in an electrostatic field," International Journal for Numerical Methods in Engineering, vol. 84, no. 13, pp. 1589-1612, 2010.

[12] L. Liu, C. Xie, B. Chen, and J. Wu, "Iterative dipole moment method for calculating dielectrophoretic forces of particleparticle electric field interactions," Applied Mathematics and Mechanics, vol. 36, no. 11, pp. 1499-1512, 2015.

[13] L. Liu, C. Xie, B. Chen, N. Chiu-On, and J. Wu, "A new method for the interaction between multiple DEP particles: iterative dipole moment method," Microsystem Technologies, vol. 22, no. 9, pp. 1-10, 2015.

[14] L. Liu, C. Xie, B. Chen, and J. Wu, "Numerical study of particle chains of a large number of randomly distributed DEP particles using iterative dipole moment method," European Journal of Mechanics B/Fluids, vol. 58, pp. 50-58, 2015.

[15] H. Feng, T. N. Wong, and Marcos, "Pair interactions in induced charge electrophoresis of conducting cylinders," International Journal of Heat and Mass Transfer, vol. 88, pp. 674-683, 2015.

[16] N. Sun and J. Y. Walz, "A model for calculating electrostatic interactions between colloidal particles of arbitrary surface topology," Journal of Colloid and Interface Science, vol. 234, no. 1, pp. 90-105, 2001. 


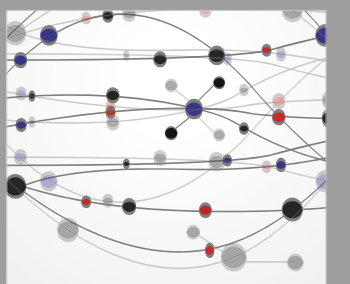

The Scientific World Journal
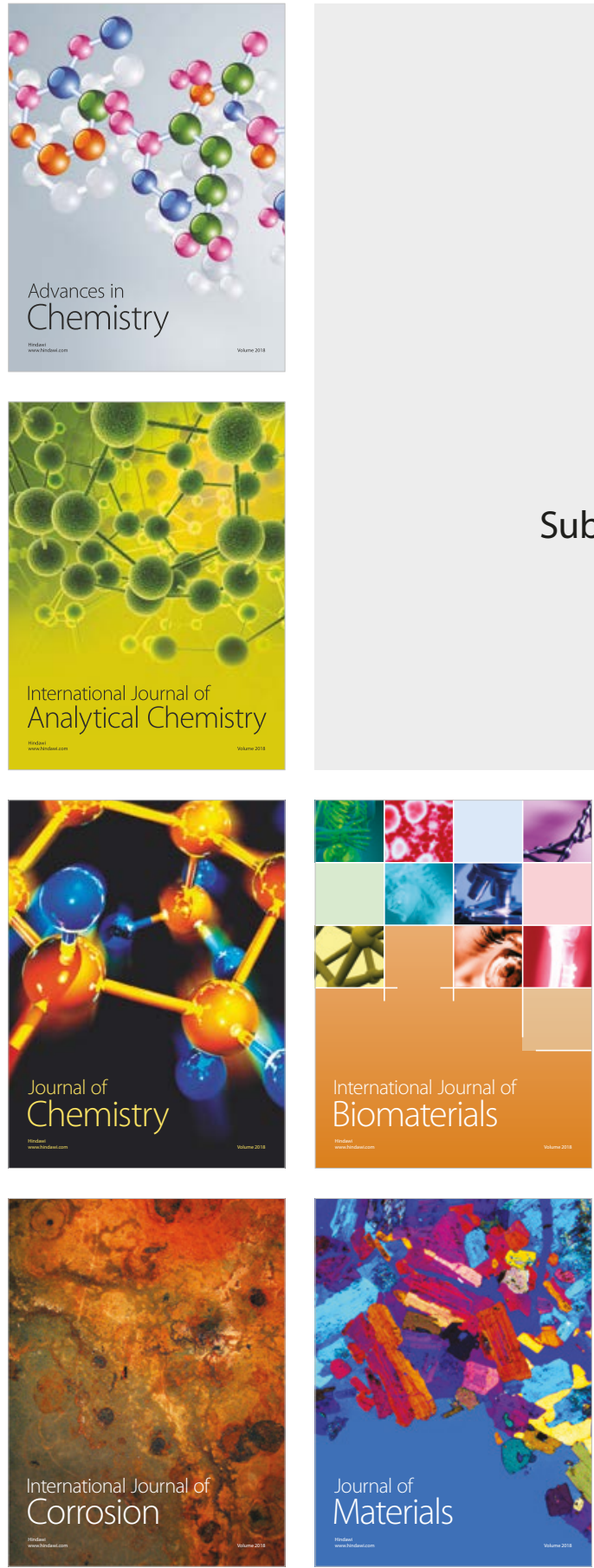

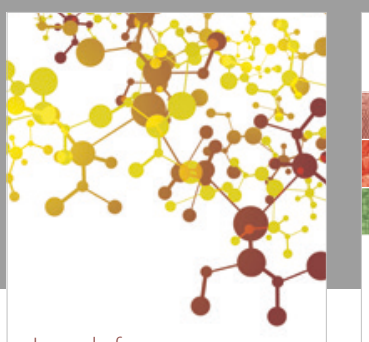

Journal of

Applied Chemistry
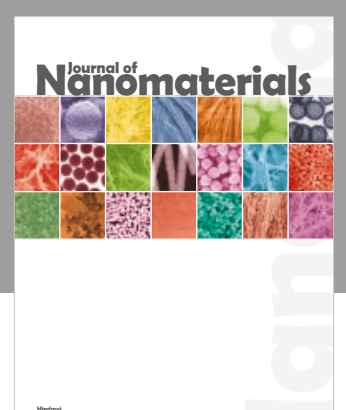

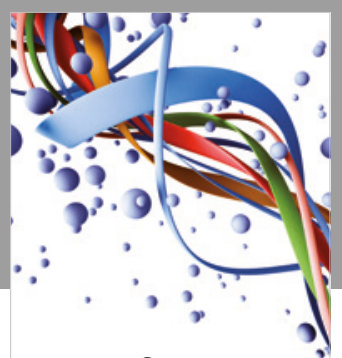

Scientifica

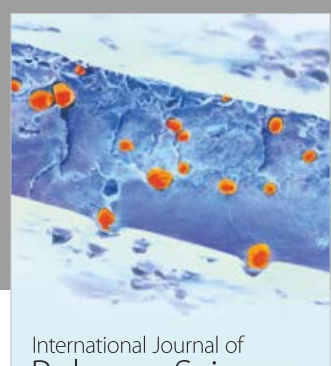

Polymer Science

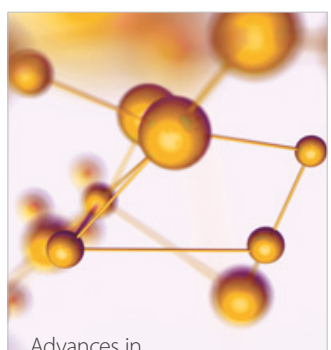

Physical Chemistry
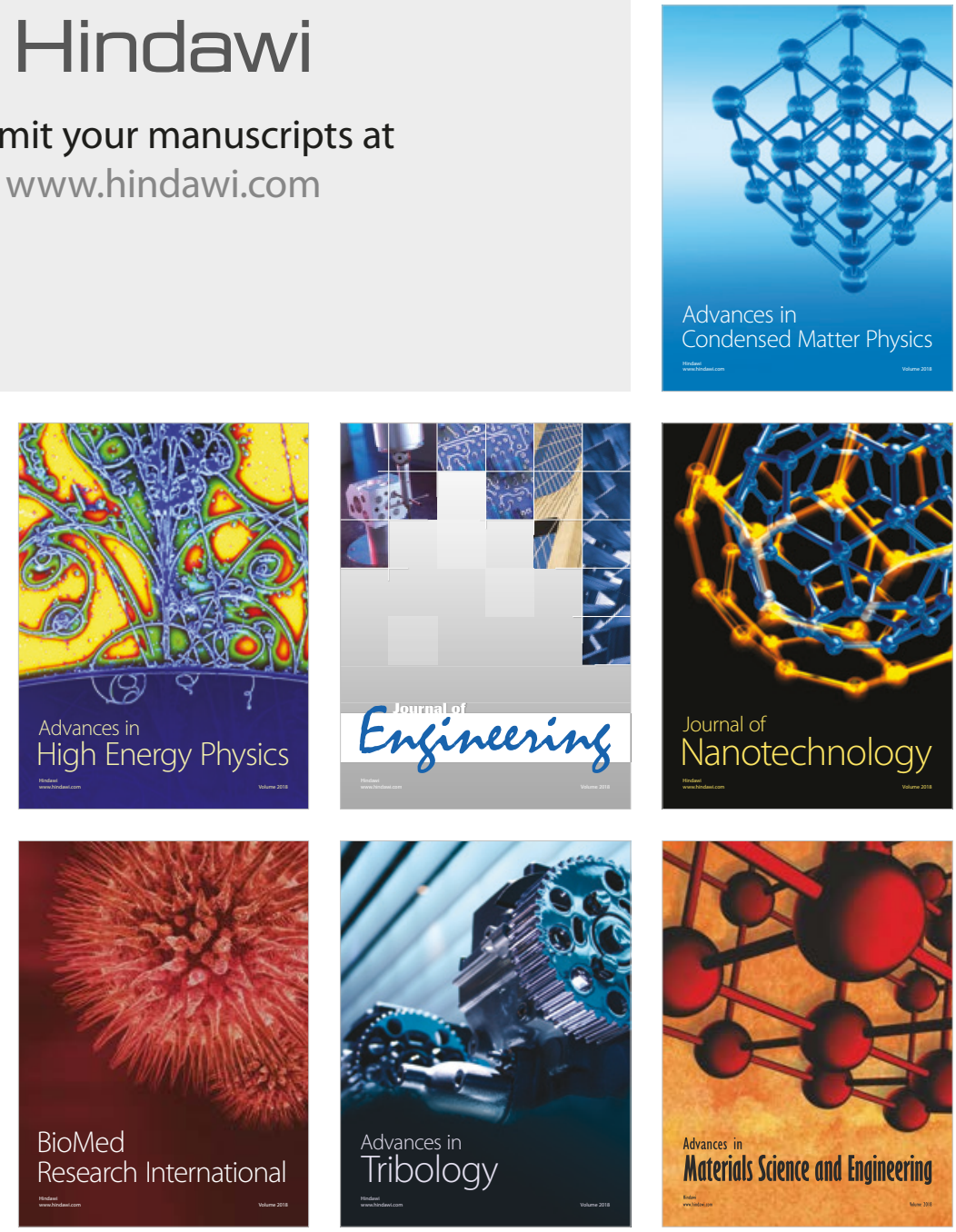